\title{
Octagonal defect lines in graphene structures
}

\author{
Wlodzimierz Jaskolski ${ }^{1}$, Marta Pelc ${ }^{1}$, Leonor Chico ${ }^{2}$ and Andres Ayuela ${ }^{3}$
}

\begin{abstract}
We study graphene nanoribbons and carbon nanotubes containing defect lines built of octagonal rings. All the calculations are performed within the $\pi$-electron tight binding approximation, taking into account electron interaction effects by means of the Hubbard model. Octagonal defect lines are the source of state localization at Fermi energy and in some cases may lead to spontaneous magnetization. We show that the localization is due to the zigzag nature of the edges of defect lines.
\end{abstract}

\section{INTRODUCTION}

Experimental techniques allow nowadays to pattern graphene into nanometer size ribbons with well controlled size and shape of the edges [1], [2]. Graphene nanoribbons (GNR) can also be obtained by unzipping carbon nanotubes (CN) [3], [4]. Either graphene ribbons or different carbon nanotubes can be connected by introducing topological defects at their interfaces. Junctions in such systems are interesting because they can reveal localized states at Fermi energy, which can influence transport and magnetic properties of these systems.

Junctions between graphene nanoribbons form onedimensional grain boundaries or defect lines. In particular, interfaces between zigzag ribbons are made of octagonal rings accompanied by pentagons. It has been shown recently [5], that such junctions can act as quasi-one-dimensional metallic wires and can yield spontaneous magnetization. Octagonal defects in graphene occur also in local divacancies [6], [7].

Junctions between carbon nanotubes are usually realized by pentagon/heptagon (5/7) topological defects. For a long time it has been commonly accepted that these defects are the source of interface states. However, we have shown [8] that interface localized states do not appear due to $5 / 7$ defects, but they originate from the zigzag edges of the joined tubes. Octagonal rings in CNs can also appear. It may happen at diagonal junctions between the tubes [9] or when unzipped tubes are clipped back after introducing topological defects at the edges.

In pure graphene structures such as ribbons or flakes the condition for the existence of edge-localized states at zeroenergy, i.e., the vanishing of the sum of the wave function coefficients at all nearest-neighbor nodes around a given node,

\footnotetext{
${ }^{1}$ W. Jaskolski and M. Pelc are with Institute of Physics, Nicolaus Copernicus University, 87-100 Torun, Poland wj at fizyka.umk.pl and martap at fizyka.umk.pl

${ }^{2}$ L. Chico is with Instituto de Ciencia de Materiales de Madrid, Cantoblanco, ES-28049 Madrid, Spain leonor.chico at icmm.csic.es

${ }^{3}$ A. Ayuela is with Centro de Fisica de Materiales, CFM-CPM CSICUPV/EHU and Donostia International Physics Center, ES-20080, San Sebsatian, Spain swxayfea at ehu.es
}

enforces localization of the corresponding wave function at only one sublattice. The appearance of topological defects yields usually mixing of graphene sublattices and therefore may disturb the formation of zero-energy localized states. This is really the case of pentagonal and heptagonal defects, which locally mix strongly the graphene sublattices [11]. Although much is known on the heptagonal and pentagonal defects, little research has been done so far concerning octagonal defects.

In this work we study several graphene systems containing such defects. We show that octagonal defects localize states at Fermi energy even if their appearance mixes both graphene sublattices. In some cases they may lead to spontaneous magnetization. We explain this localization using simple rules presented in Ref. [12]. All the calculations are performed within the $\pi$-electron tight binding (TB) approximation. The electron interaction effects are taken into account by means of the Hubbard model.

\section{GRAPHENE RIBBONS AND CARBON NANOTUBES WITH OCTAGONAL DEFECT LINES}

During the last few years several experimental and theoretical works [5], [6], [7] have been devoted to graphene ribbons and carbon nanotubes containing defect lines built as periodic sequence of one octagon and two pentagons, see Fig. 1 (b). Here we investigate the role of octagons in the formation of localized states at the Fermi energy, that appear in such systems.

In our recent paper [12] we investigated edge-localized states in graphene nanoribbons. In that work we started from pure zigzag ribbons and by means of a construction technique, i.e., adding and connecting edge nodes, we worked out a very general rule governing the existence of localized states at arbitrary edges. For the present study we use a similar methodology in order to explore the electronic states localized at defect lines containing octagons.

We start with a zigzag GNR with a defect line made of octagons only, depicted in Fig 1 (a). Pentagons, as shown in Fig. 1 (b), are introduced at a later stage by connecting every second octagon. Additionally, we consider also rolledup systems to get rid of zigzag-edge localized states. We will study the differences among these closely-related structures in order to elucidate the role of topological defects in their electronic properties.

\section{A. Defect lines made of successive octagons}

We first consider zigzag GNR with a defect line made of octagons only, as shown in Fig 1 (a). The unit cell of the investigated ribbon, shown in Fig. 1 (a), contains 
a)

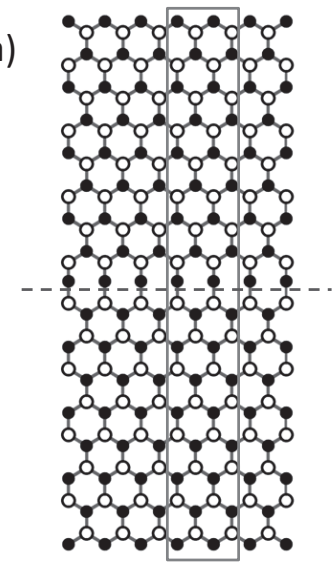

b)

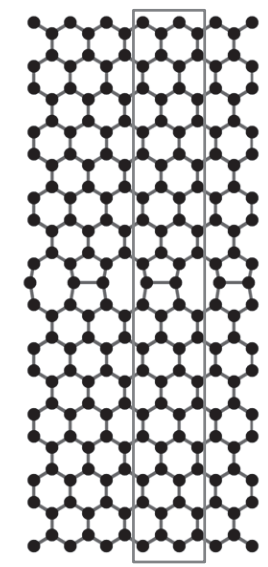

Fig. 1. Zigzag graphene nanoribbon (ZGNR) with a defect line composed of (a) consecutive octagons and (b) octagons with an adjacent pair of pentagons. The ZGNR extends horizontally. Filled and empty circles in (a) represent different graphene sublattices. Rectangles mark the unit cells of the ribbons. The dashed horizontal line in (a) marks a cutting line, which separates the ribbon into two ZGNRs, one of them having a Klein-like edge at one side.

58 atoms. It has been chosen to be the same as in Ref. [5]. Such ribbon can be seen as two connected zigzag GNRs, but one of them having Klein-like edge [12] at one side, as shown in Fig. 1 (a). This structure system is less stable than the experimentally achieved one [5], in which every second octagon reconstructs into a pair of pentagons (see Fig. 1(b)). However, this is an ideal starting point system, helpful for understanding of the localization effects at octagonal defects. Notice that this system can be divided in two bipartite lattices. Consequently, the electronic spectrum should preserve electron-hole symmetry, as can be seen in the energy bands shown in Fig. 2 (a). Although there is not sublattice mixing, the defect line makes both zigzag edges belong to the same sublattice, differently to a defect-free nanoribbon. This introduces an imbalance between atoms belonging to opposite sublattices, with an excess of two atoms in that of the edge atoms. Thus, due to Lieb's theorem, there should be a net magnetic moment of 2 Bohr magnetons in this system, as we will show below.

The wave functions corresponding to four zero-energy bands at $k=0$ are presented in panel (b) of Fig. 2. The ones labeled by $Z_{e}$ belong to two states localized at the outer (external) zigzag edges of the ribbon. The other two flat bands extending over the entire Brillouin zone have their wave functions localized at the octagonal defects, but in opposite sublattices. One of them looks similar to a state localized at the Klein edge[12] (labeled $K$ ), while the other reminds of the edge states localized in zigzag nanoribbons (labeled $Z_{i}$ ), but separated by the Klein atoms forming part of the octagons. Note that these Klein atoms have only two nearest neighbors, i.e., coordination number equal to 2 . The fourth wave function shown in Fig. 2 (b) is localized at the atoms close to the Klein nodes. They are like zigzaglike edge states, but separated by the extra row of Klein atoms, where the wavefunction has zero weight. As the row

of Klein atoms is in fact shared by both inner zigzag edges, the electrons are shared by these two zigzag-like rows of atoms, similar to a resonant state composed by a mixture of a double and a single bond in an aromatic ring. This idea is corroborated by the fact that the maximum of the wave function at these nodes is half of the maximum value of three other wave functions, so it can be considered that the state is shared between the two inner zigzag edges.
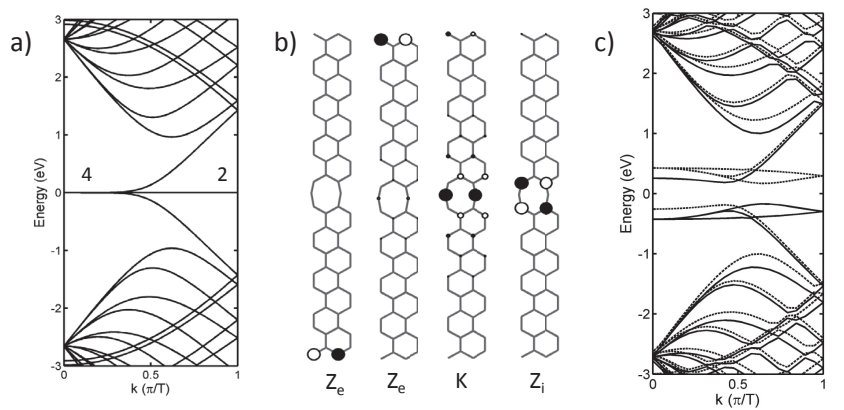

Fig. 2. (a) Energy bands of a ZGNR with a defect line composed of octagons. The wave vector $k$ is given in units of $\pi / \mathrm{T}$, where $\mathrm{T}$ is the length of the unit cell. The degeneracies of $E=0$ bands at $k=0$ and $k=\pi$ are indicated in the Figure. (b) Wave functions corresponding to four zero-energy bands at $k=0$. Black and white dots represent positive and negative signs of the wave function. (c) Energy spectrum calculated including electron-electron interactions by means of a Hubbard model. Solid lines denote spin up bands; dotted lines show the spin down bands. The Fermi energy is at $0 \mathrm{eV}$.

As we expect a net magnetization in this system, we consider electron-electron interaction effects by employing the Hubbard model, in which the on-site Coulomb repulsion is included. The resulting bands are presented in Fig. 2 (c). The solid lines correspond to spin-up (majority) bands and the dotted lines represent spin-down (minority) solutions. At $k$ close to zero and for energies below the Fermi level, there are three nearly degenerated spin-up bands and one spindown band. All the corresponding spin-up wave functions are localized at the same sublattice: one at the bottom zigzag edge, the second at the upper zigzag edge and the third one at the internal Klein nodes of the octagonal defect. They very much resemble the first three TB functions shown in Fig. 2 (b). The wave function of the spin-down band is localized at the other nodes at the octagon, i.e., at the complementary sublattice. At $k>2 / 3$, there are two spin-up bands below $E_{F}$, while their spin-down counterparts are unoccupied. As a result, the spin polarization per unit cell is close to $2 \mu_{B}$ and the system reveals spontaneous magnetization, consistent with the number given by the Lieb's theorem for this system.

Let us now roll up the system to get an armchair carbon nanotube with the octagonal line of defects along the tube axis. This way we just get rid of the zigzag outer edges and their corresponding localized states. Note that, since we have to connect the ZGNR edge nodes which belong to the same sublattice, the resulting nanotube does not have globally defined (i.e., valid throughout the entire tube) atom sublattices. The corresponding band structure and wave functions of the zero energy states at $k=0$ are shown in 
Fig. 3. It is clear from this Figure that, due to the sublattice mixing, these bands do not have electron-hole symmetry. Since this system does not have zigzag edges, the two edge-
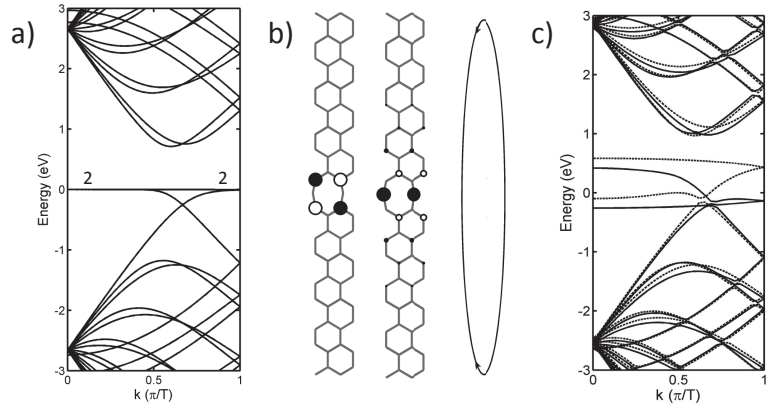

Fig. 3. (a) Energy bands of the armchair carbon nanotube with a defect line composed of octagons obtained by rolling up a ZGNR with octagonal defects. (b) Wave functions corresponding to two zero-energy bands at $k=0$. The way of rolling-up the ZGNR unit cell is indicated. (c) Energy spectrum of the same system calculated with a Hubbard model. Solid lines: spin-down bands, dotted lines: spin-up bands.

localized states present in the related ribbon disappear. The two surviving zero-energy bands are related to the line of octagonal defects and, in fact, the wavefunctions at $k=0$ look exactly the same as the corresponding defect-related wavefunctions in the unrolled system, as it can be seen in 3 (b). This indicates that close to the octagons the graphene sublattices are locally well defined.

Moreover, there is a small energy gap between the flat band at the Fermi energy and the conduction bands, which is unusual for armchair tubes. However, the Fermi energy is slightly above the crossing point at $k=2 / 3$ and it does not enter into the gap, so the nanotube preserves its metallic character. Nevertheless, the electrons around the Fermi energy mainly occupy the states around the Klein nodes. It is important to emphasize that the robust metallicity of armchair nanotubes is strongly disturbed because the line of defects perturbs the nanotube wavefunctions, which in principle are delocalized around the circumference.

The bandstructure calculated within the Hubbard model is presented in Fig. 3 (c). As for this system Lieb's theorem does not apply, the magnetic moment has to be obtained from the electronic spectrum. For $k<2 / 3$ the two flat bands just below $E_{F}$ have different spin polarization. They are both localized at the octagonal defect, but each one at a different local sublattice, as the TB wave functions in Fig. 3 (b). However, for $k>2 / 3$ the couple of occupied flat bands below the Fermi energy have the same spin polarization. As a result, the magnetization is almost three times weaker than in the previous case, i.e., equals $\sim 0.6 \mu_{B}$.

\section{B. Alternating octagons between pairs of pentagons}

Let us now consider a system that has been recently studied both experimentally and theoretically, namely a ZGNR with a defect line that alternate octagons and pairs of pentagons. This geometrical reconstruction of octagons into pentagons is driven by the $\sigma$ electrons, but they do not need to be considered as they lie at much lower energies. Because of the reconstruction, the atoms which were identified as Klein-node like have now three nearest-neighbors, i.e., have a coordination number 3,[5], [13], [14] as shown in Fig. 1 (b). Due to the presence of the pentagons there is a local sublattice mixing at the row of defects. The band structure for the unrolled system is shown in Fig. 4 (a).
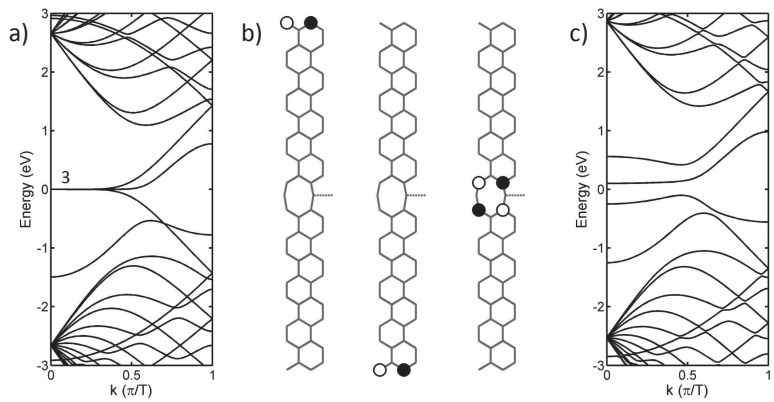

Fig. 4. (a) Band structure of a ZGNR with a defect line composed of octagons and pentagons as depicted in Fig.1 (b). (b) Wave functions corresponding to the three zero-energy bands in the TB calculation at $k=0$. Black and white dots represent different signs of the TB wave function. (c) Band structure for the same system calculated within the Hubbard model. Spin-down and spin-up bands are degenerate in energy.

As expected, the previous state localized at the bonds between two octagons moves well away from the Fermi energy due to the newly formed bond. This is because of the strong sublattice mixing, which occurs locally due to the pentagons. However, the other state does not change much. As its wavefunction has null coefficients at the newly connected nodes, this state is not affected by the formation of the pentagons. It is noteworthy that the corresponding band is much less affected by the on-site Coulomb interaction than the states localized at the GNR edges, which split up and down, away from zero energy due to the electronic repulsion, as it can be seen in the bands calculated within the Hubbard model, shown in Fig. 4(c). The flattest band at Fermi energy is localized at the octagon for $k=0$. Our results around the Fermi energy coincide with those shown in Ref. [15], obtained with a density functional approach. The outer edges allow for localization of both the spindown and the spin-up functions at the same nodes without significant energy splitting between the corresponding bands, which is typical for zigzag nanoribbons. The flattest band at the Fermi energy, i.e., the one localized at octagons, is also a resonance between the two states belonging to the so-called inner zigzag edges, as shown for the pure octagon cases, and therefore it is not affected much by the connection of the Klein-like modes.

Let us finally consider the nanotube with the double pentagon-octagon defect line. By rolling up the ribbon with the defects we obtain an armchair nanotube with a line of defects along the tube axis. Such nanotube was studied by Okada and coworkers for the first time[16]. As we are connecting the zigzag edges, the corresponding localized states disappear and only one flat band for $k<2 / 3$ survives 
in the vicinity of the Fermi energy, as depicted in Fig. 5 (a). The TB wave function corresponding to this flat band is shown in Fig. 5 (b), and it is localized at the octagon. As in the related ribbon structure, the inclusion of the on-site Coulomb term in the Hubbard model [Fig. 5 (c)] changes the spectrum negligibly.
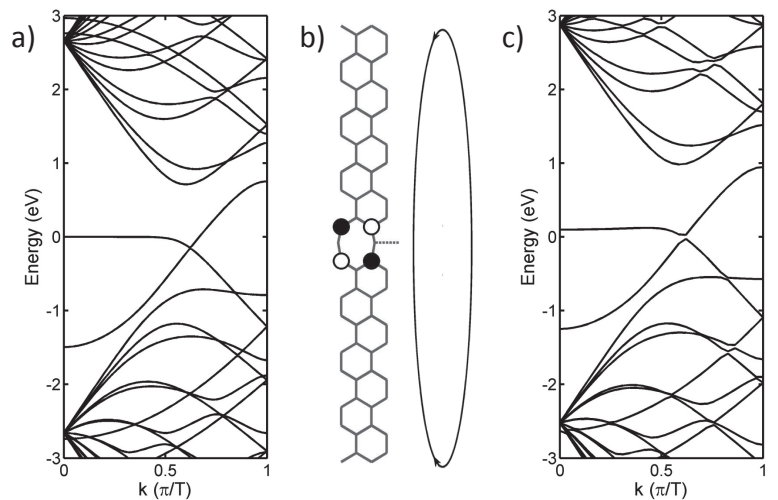

Fig. 5. (a) Band structure of an armchair nanotube (rolled-up ZGNR) with a defect line composed of alternating octagons and pairs of pentagons calculated with the TB approximation. (b) Wavefunction of the flat band localized at the octagon defect. (c) Bandstructure of the armchair nanotube with the double pentagon-octagon defect line calculated within the Hubbard model.

\section{Explanation of the results using rules of Ref. [12]}

In Ref. [12] we presented a series of hybridization rules based on a construction technique which allow for a simple explanation of the existence and degree of degeneracy of zero-energy flat bands localized in graphene ribbons with arbitrary edge shape. The rules are based on(a) the Brillouin zone folding and (b) splitting of degenerated flat bands localized at different graphene sublattices whenever their wave functions overlap, that is, when they are connected by a nearest-neighbor hopping. Notice that in some of the systems shown here, the lattice is no longer bipartite, so the criterion will be whether a nearest-neighbor hopping is set between atoms with a non-negligible wavefunction amplitude.

Here we show how simply those rules explain all the results obtained in the previous sections. The construction technique is shown in Fig. 6. We start from two zigzag GNRs, which result from horizontally cutting the ribbon shown in Fig. 1 (a) slightly below the line of the central nodes. This yields two ZGNRs, one of them having the Klein edge atoms at one side. Since we will later discuss ZGNR or CNTs with line of defects composed of octagon and two pentagons, we consider unit cell twice wider than the usual cell of zigzag ribbons, thus performing an extra folding of the bands. Schematic band structures for such two ribbons are shown in the panel (a) of Fig. 6. Shaded areas represent band continua for the case of very wide ribbons. The symbols $\mathrm{Z}_{e}, \mathrm{Z}_{i}$ and $K$ mark the zero-energy states that localize at outer (external), internal and Klein edges of ZGNRs, respectively. When both ribbons are again connected, no splitting of the zero-energy bands occurs, because all the (a)

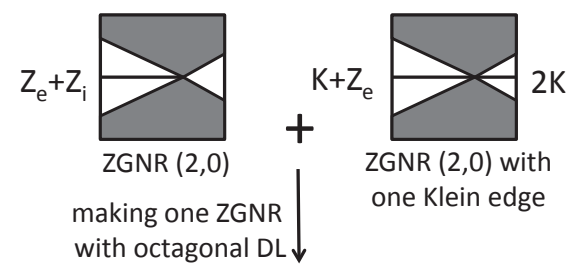

(b)

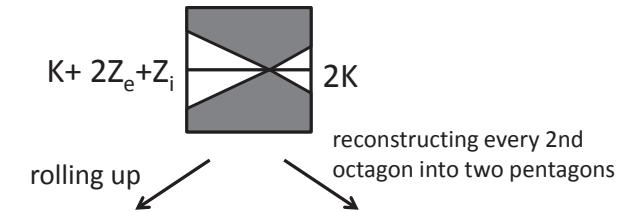

(cl)
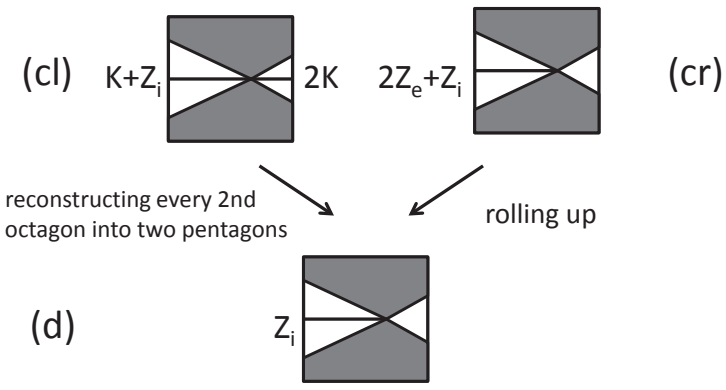

Fig. 6. Schematic bands structures close to Fermi energy for the systems investigated in Section II. (a) Two independent ZGNR, one of them having Klein edges at one side (right). Band structures shown in the folded BZ (b) Two ZGNRs connected to form a ZGNR with a line of defects made of octagons only. (cl) Rolled up ZGNR, (cr) ZGNR with the Klein nodes connected. (d) Armchair nanotube with line of defects composed of octagons and pentagons. $\mathrm{Z}_{e}$ marks states localized at outer zigzag edges, $\mathrm{Z}_{i}$ marks state localized at internal zigzag edges (at the octagon), and $\mathrm{K}$ represents Klein edge-localized states. Rolling up ZGNR or bonding Klein nodes is represented by the arrows.

states are either localized at farther than nearest-neighbor atoms or in different regions of the ZGNR. This is visualized in panel (b) of Fig. 6.

When we roll up the system, see panel (cl), we eliminate the edges, so we have to remove two $\mathrm{Z}_{e}$ bands from the spectrum. The resulting band structure close to Fermi energy looks the same as in Fig. 3 (a). On the other hand, if instead of rolling up the system, we reconstruct every second octagon into a pair of pentagons, we strongly mix locally the graphene sublattices. The states which were localized at the Klein nodes hybridize, so the corresponding bands split and merge into the continuum. This is shown in panel (cr); the resulting spectrum has the same appearance as the band structure shown in Fig. 4 (a).

Finally, we consider an armchair nanotube having a defect line made of octagons and double pentagons. Independently on whether we start from the tube with octagonal defects only [panel (cl)] or from the ZGNR with a defect line composed of octagons and pentagons [panel (cr)], we end up with the band structure shown in panel (d). In both cases one has to remove either the edge bands or the bands localized at the Klein nodes. The resulting spectrum has the same features as the band structure presented in Fig. 5 (a). Note that the degeneracies of the zero-energy bands presented in 
panels (b), (cl), (cr) and (d) is the same as in panels (a) of Figures 2-5, respectively.

Let us summarize these results. By making extra bonds, the states hybridize, so the bands split and move to the continuum. Firstly, by rolling the nanoribbons we eliminate the outer zigzag states. Secondly, by bonding the Klein nodes at alternating octagons, the related state disappears and we are left with the resonant zigzag-like state localized at the octagons.

\section{SUMMARY AND CONCLUSIONS}

We have investigated graphene nanoribbons and carbon nanotubes containing defect lines made of successive octagons or octagons accompanied by pentagons. We have shown that octagonal defects lines in graphene structures lead to state localization with energies close to the Fermi level. The appearance of localized flat bands can be easily explained using simple rules and diagrams introduced in our previous work [12]. We show that one of the localized states is a resonance between two states belonging to the inner zigzag edges confining the line of defects. The localization happens independently on the sublattice mixing, which takes place when octagons are accompanied by pentagons. Such defects may therefore indicate reactivity sites in defected graphene. Using the Hubbard model we have investigated also the on-site electron-electron interaction effects and we have shown that their influence on the localized zero-energy states depends on particular case. The spin-up and spin-down bands split only when there is no sublattice mixing at the nodes, where they are localized. We have shown that such structures may reveal spontaneous magnetization.

\section{ACKNOWLEDGMENT}

This work was supported by the Polish National Science Center (OPUS2/ST3 Grant), the Basque Government through the NANOMATERIALS project (Grant No. IE05151) under the ETORTEK Program (iNanogune), the Spanish Ministerio de Ciencia y Tecnología (Grant Nos. TEC200768065-C03-03 and FIS2007-66711-C02-02, FIS2009-08744, and MONACEM projects), and the University of the Basque Country (Grant No. IT-366-07). A.A. and L.C. acknowledge the Nicolaus Copernicus University of Torun for generous hospitality during their visits. M.P. acknowledges support from the Faculty of Physics, Astronomy and Informatics, NCU.

\section{REFERENCES}

[1] X. Jia, M. Hofmann, V. Meunier, B. G. Sumpter, J. Campos-Delgado, J. M. Romo-Herrera, H. Son, Y.-P. Hseih, A. Reina, J. Kong, M. Terrones, and M. S. Dresselhaus, Controlled formation of sharp zigzag and armchair edges in graphitic nanoribbons, Science 323, 1701, 2009.

[2] X. Wang and $\mathrm{H}$. Dai, Etching and narrowing of graphene from the edges, Nature Chemistry 2, 661, 2010.

[3] D. V. Kosynkin, A. L. Higginbotham, A. Sinitskii, J. R. Lomeda, A. Dimiev, B. Price, and J. M. Tour, Longtitudional unzipping of carbon nanotubes to form graphene nanoribbons, Nature 458, 872, 2009.

[4] L. Jiao, L. Zhang, X. Wang, G. Diankov, and H. Dai, Narrow graphene nanoribbons from carbon nanotubes, Nature 458, 877, 2009.

[5] J. Lahiri, Y. Lin, P. Bozkurt, I. Oleynik, and M. Batzill, An extended defect in graphene as a metallic wire, Nature Nanotech. 5, 326, 2010.
[6] M. M. Ugeda, I. Brihuega, F. Hiebel, P. Mallet, J.-Y. Veuillen, J. M. Gomez-Rodriguez, and F. Yndurain, Electronic and structural characterization of divacancies in irradiated graphene, Phys. Rev. B 85, 121402, 2012.

[7] Y. Kim, J. Ihm, E. Yoon, and G.-D. Lee, Dynamics and stability of divacancy defects in graphene, Phys. Rev. B 84, 075445, 2011.

[8] H. Santos, A. Ayuela, W. Jaskolski, M. Pelc, and L. Chico, Interface States in Carbon Nanotube Junctions: Rolling up Graphene, Phys. Rev. B 80, 035436, 2009.

[9] W. Jaskolski, M. Pelc, H. Santos, L. Chico, and A. Ayuela, Interface Bands in Carbon Nanotube Superlattices, phys. stat. sol. C 7, 382, 2010.

[10] J. N. B. Rodrigues, P. A. D. Gancalves, N. F. G. Rodrigues, R. M. Ribeiro, J. M. B. Lopez de Santos, and R. N. M. Peres, Zigzag graphene nanoribbon edge reconstruction with Stone-Wales defects, Phys. Rev. B 84, 155435, 2011.

[11] The only exception is the $5 / 7$ reconstructed zigzag edge, where $E=0$ states appear in TB approach due to a special symmetry[10].

[12] W. Jaskolski, A. Ayuela, L. Chico, M. Pelc, and H. Santos, Edge states and flat bands in graphene nanoribbons with arbitrary geomatries, Phys. Rev. B 83, 235424, 2011.

[13] L. Kou, Ch. Tang, W. Guo, and Ch. Chen, Tunable magnetism in strained graphene with topological line defekt, ACS Nano 5, 1012, 2011.

[14] D. J. Appelhans, L. D. Carr, and M. T. Lusk, Embedded ribbons of graphene allotropes: an extended defect perspective, New J. Phys. 12, 125006, 2010.

[15] X. Lin and J. Ni, Half-metallicity in graphene nanoribbons with topological line defects, Phys. Rev. B 84, 075461, 2011.

[16] S. Okada, K. Nakada, K. Kuwabara, K. Daigoku, and T. Kawai, Ferromagnetic spin ordering on carbon nanotubes with topological line defects, Phys. Rev. B 74, $121412,2006$. 